

L. Rüschendorf, W. Thomsen, Closedness of sum spaces and the generalized Schrödinger problem, Теория вероятн. и ее примен., 1997, том 42, выпуск 3, 576-590

DOI: https://doi.org/10.4213/tvp1955

Использование Общероссийского математического портала Math-Net.Ru подразумевает, что вы прочитали и согласны с пользовательским соглашением http://www.mathnet.ru/rus/agreement

Параметры загрузки:

IP : 54.198 .64 .247

26 апреля 2023 г., 18:09:49

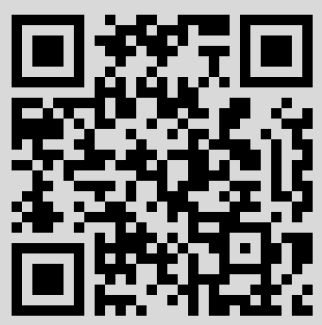


(C) 1997 г. RÜSCHENDORF L. ${ }^{*}$, THOMSEN W. ${ }^{* *}$

\title{
CLOSEDNESS OF SUM SPACES AND THE GENERALIZED SCHRÖDINGER PROBLEM
}

\begin{abstract}
Установлены некоторые общие свойства замкнутости сумм пространств измеримых функцй. В качестве применения доказаны существование и единственность решений обобщенной задачи ІІредингера при некотором условии интегрируемости, но без каких-либо предположений о топологии или ограниченности. Полученные свойства позволяют также доказать интересный результат о структуре законов с многомерными частными распределениями, установить существование оптимальных аппроксимаций в аддитивных статистических моделях и обобщить представление Колмогорова для непрерывных функций нескольких переменных. Из последнего результата вытекает, что любая локально ограниченная измеримая функция имеет точное представление нейронной сетью с одним скрытым слоем.
\end{abstract}

Ключевые слова и фразы: уравнение IІредингера, сумма пространств, аддитивные модели, многомерные частные распределения, представление Колмогорова.

\section{Introduction}

The main motivation of this paper is to derive existence and uniqueness results for some generalized versions of the Schrödinger equations. It turns out that these results are based on some crucial closedness properties of sum spaces of measurable functions, which are derived in Section 2. These closedness properties also allow to prove some interesting existence results for projections in additive statistical models, furthermore, to develop an interesting structural property of distributions with multivariate marginals, and to derive an extension of Kolmogorov's famous representation theorem for continuous functions of $n$-variables.

Let $\mu$ be a probability measure on a product space $(E, \mathcal{A})=\left(E_{1}, \mathcal{A}_{1}\right) \otimes$ $\left(E_{2}, \mathcal{A}_{2}\right)$ with marginals $\mu_{i}$ on $E_{i}$. Let $L^{0}\left(E_{i}, \mathcal{A}_{i}, \mu_{i}\right)=L^{0}\left(\mu_{i}\right)$ denote the class of $\mathcal{A}_{i}$-measurable real functions. It is clear from the context, if $f \in$ $L^{0}\left(\mu_{i}\right)$ stands for some version or for the equivalence-class $\bmod \mu_{i}$-nullfunctions. Furthermore, let $\nu_{i} \in M^{1}\left(E_{i}, \mathcal{A}_{i}\right)$ be probability measures on

\footnotetext{
*Institut für Mathematische Stochastik, Eckerstr. 1, 79104 Freiburg, Germany.

${ }^{* *}$ Institut für Mathematische Statistik, Einsteinstr. 62, 48149 Münster, Germany.
} 
$\left(E_{i}, \mathcal{A}_{i}\right)$ continuous with respect to $\mu_{i}$ with densities $r_{i}=d \nu_{i} / d \mu_{i}$. Consider the following system of nonlinear integral equations which we denote as (generalized) Schrödinger-problem:

$$
\begin{array}{ll}
E_{\mu}\left(a \otimes b \mid \pi_{1}=x\right)=r_{1}(x) & \mu_{1} \text {-a.e. } \\
E_{\mu}\left(a \otimes b \mid \pi_{2}=y\right)=r_{2}(y) & \mu_{2} \text {-a.e., }
\end{array}
$$

where $a \otimes b \in L_{+}^{0}(\mu), a \otimes b(x, y):=a(x) b(y)$, and $\pi_{i}: E_{1} \times E_{2} \longrightarrow E_{i}$ are the projections on $E_{i}$.

If $\mu$ has a density $h$ with respect to $\mu_{1} \otimes \mu_{2}$, i.e., $\mu=h \mu_{1} \otimes \mu_{2}$, then (1.1) is equivalent to

$$
\begin{array}{ll}
\int a(x) b(y) h(x, y) \mu_{1}(d y)=r_{1}(x) & \mu_{1} \text {-a.e. } \\
\int a(x) b(y) h(x, y) \mu_{2}(d x)=r_{2}(y) & \mu_{2} \text {-a.e. }
\end{array}
$$

Equation (1.1) is equivalent to the following marginal problem. Let $M\left(\nu_{1}, \nu_{2}\right)$ denote the set of all probability measures on $(E, \mathcal{A})$ with marginals $\nu_{1}, \nu_{2}$. Then (1.1) is equivalent to:

Find $a \otimes b \in L_{+}^{0}(\mu)$ such that

$$
\nu=a \otimes b \mu \in M\left(\nu_{1}, \nu_{2}\right)
$$

Problem (1.1), (1.3) is of interest for the structure of (generalized) Schrödinger bridges (cf. [2], [3], [4], [6], [7], [11], [15], [21]). In our previous paper [18] we proved the existence and uniqueness of solutions of (1.2) with $a \in L_{+}^{0}\left(\mu_{1}\right), b \in L_{+}^{0}\left(\mu_{2}\right)$ under an integrability condition but without any assumptions on the boundedness of $h$ or topological restrictions extending related results in [1], [8], [10], [11] and [20]. In this paper we investigate the general problem (1.1), (1.3). The progress is established by some crucial closedness properties of sum spaces of measurable functions in Section 2 which need the development of some new tools, and which also find some further applications in Sections 3 and 4. In particular we consider applications to optimal approximations in additive statistical models, some structure results for distributions with given marginals and a «generalization» of Kolmogorov's representation theorem to measurable functions. A part of the problems is caused by the possibly complicated structure of the support of $\mu$.

\section{Closedness Properties of Sum Spaces}

Consider the sum space of measurable functions

$$
F=L^{0}\left(\mu_{1}\right) \oplus L^{0}\left(\mu_{2}\right)=\left\{\varphi=f \oplus g ; f \in L^{0}\left(\mu_{1}\right), g \in L^{0}\left(\mu_{2}\right)\right\},
$$

5 Теорих вероятностей и ее применения, № 3 
where $f \oplus g(x, y)=f(x)+g(y) . F$ is a subspace of

$$
G=\left(M\left(E_{1}\right) \oplus M\left(E_{2}\right)\right) \cap L^{0}(\mu),
$$

where $M\left(E_{i}\right)$ is the class of all real valued functions (maps) on $E_{i}$ and $L^{0}(\mu)=L^{0}\left(E_{1} \times E_{2}, \mathcal{A}_{1} \otimes \mathcal{A}_{2}, \mu\right)$ is supplied with the complete metric of $\mu$-stochastic convergence. In this section we are concerned with a characterization of the closure of $F$ in $L^{0}(\mu)$ with respect to $\mu$-stochastic convergence (and related types of convergence). The following result is obtained by using essentially methods of the proofs in [18].

Proposition 2.1. $G$ is closed in $L^{0}(\mu)$ with respect to $\mu$-stochastic convergence. In particular, the closure of $F$ in $L^{0}(\mu)$ is a subset of $G$.

$\mathrm{P}$ r o of. 1. For any limit point $\varphi=\varphi(x, y)$ of $G$ with respect to $\mu$-stochastic convergence, there exists a sequence $f_{n} \oplus g_{n}$ in $G$ such that

$$
f_{n}(x)+g_{n}(y) \longrightarrow \varphi(x, y), \quad \mu \text {-a.e. }
$$

Then $C:=\left\{(x, y) \in E ; \lim _{n}\left(f_{n}(x)+g_{n}(y)\right)\right.$ exists $\} \in \mathcal{A}_{1} \otimes \mathcal{A}_{2}, \mu(C)=1$ and without loss of generality

$$
\varphi(x, y)=\lim _{n}\left(f_{n}(x)+g_{n}(y)\right) 1_{C}(x, y) .
$$

2. Choose $\left(x_{1}, y_{1}\right) \in C$ and define

$$
\begin{aligned}
f_{n}^{1}(x) & :=f_{n}(x)-f_{n}\left(x_{1}\right), \quad g_{n}^{1}(y):=g_{n}(y)+f_{n}\left(x_{1}\right), \\
C_{1}^{1} & :=\left\{x \in E_{1} ; f^{1}(x):=\lim _{n} f_{n}^{1}(x) \text { exists }\right\}, \\
C_{2}^{1} & :=\left\{y \in E_{2} ; g^{1}(y):=\lim _{n} g_{n}^{1}(y) \text { exists }\right\} .
\end{aligned}
$$

Then $\left(x_{1}, y_{1}\right) \in C_{1}^{1} \times C_{2}^{1}$ and for $(x, y) \in C_{1}^{1} \times C_{2}^{1}$ it holds

$$
f_{n}(x)+g_{n}(y)=\left(f_{n}(x)-f_{n}\left(x_{1}\right)\right)+\left(g_{n}(y)+f_{n}\left(x_{1}\right)\right) \longrightarrow f^{1}(x)+g^{1}(y),
$$

i.e., $(x, y) \in C$. Therefore, $C_{1}^{1} \times C_{2}^{1} \subset C$.

3. Choose $\left(x_{2}, y_{2}\right) \in C \backslash C_{1}^{1} \times C_{2}^{1}$ and define $f_{n}^{2}, g_{n}^{2}, f^{2}, g^{2}, C_{1}^{2}, C_{2}^{2}$ as in 2. with $\left(x_{1}, y_{1}\right)$ replaced by $\left(x_{2}, y_{2}\right)$. Then

$$
C_{1}^{1} \cap C_{1}^{2}=\varnothing, \quad C_{2}^{1} \cap C_{2}^{2}=\varnothing .
$$

To prove (2.5) let $x \in C_{1}^{1} \cap C_{1}^{2}$. Then $f_{n}(x)-f_{n}\left(x_{1}\right) \longrightarrow f^{1}(x)$ and $f_{n}(x)-f_{n}\left(x_{2}\right) \longrightarrow f^{2}(x)$ and, therefore,

$$
f_{n}\left(x_{2}\right)-f_{n}\left(x_{1}\right) \longrightarrow f^{1}(x)-f^{2}(x)=f^{1}\left(x_{2}\right) .
$$

This implies that $x_{2} \in C_{1}^{1}$. From

$$
\begin{aligned}
g_{n}\left(y_{2}\right)+f_{n}\left(x_{1}\right) & =\left(g_{n}\left(y_{2}\right)+f_{n}\left(x_{2}\right)\right)-\left(f_{n}\left(x_{2}\right)-f_{n}\left(x_{1}\right)\right) \\
& \longrightarrow g^{2}\left(y_{2}\right)-f^{1}\left(x_{2}\right)
\end{aligned}
$$


it follows by (2.3) that $y_{2} \in C_{2}^{1}$. This contradicts $\left(x_{2}, y_{2}\right) \notin C_{1}^{1} \times C_{2}^{1}$. By (transfinite) induction we obtain a (possibly uncountable) family $\left(C_{1}^{i} \times\right.$ $\left.C_{2}^{i}, i \in I\right)$, such that $\left(x_{i}, y_{i}\right) \in C_{1}^{i} \times C_{2}^{i}, C_{1}^{i} \cap C_{1}^{j}=\varnothing, C_{2}^{i} \cap C_{2}^{j}=\varnothing$ for all $i, j \in I, i \neq j$, and $C=\sum_{i \in I} C_{1}^{i} \times C_{2}^{i}$. Furthermore, as in point 2 we obtain functions $f^{i} 1_{C_{1}^{i}}$ and $g^{i} 1_{C_{2}^{i}}$ such that

$$
\varphi(x, y) 1_{C}(x, y)=(f(x)+g(y)) 1_{C}(x, y),
$$

where $f(x):=\sum_{i \in I} f^{i}(x) 1_{C_{1}^{i}}(x)$ and $g(y):=\sum_{i \in I} g^{i}(y) 1_{C_{2}^{i}}(y)$.

R e m a r k s. a) Additionally, we point out that the components $C_{1}^{i} \times$ $C_{2}^{i}$ of the convergence set $C=\sum_{i \in I} C_{1}^{i} \times C_{2}^{i}$ are in $\mathcal{A}_{1} \otimes \mathcal{A}_{2}$. Indeed, $C_{x_{i}}=C_{2}^{i}$, where $C_{x_{i}}$ denotes the $x_{i}$-section of $C \in \mathcal{A}_{1} \otimes \mathcal{A}_{2}$, hence

$$
C_{2}^{i} \in \mathcal{A}_{2} \quad \text { and } \quad C_{1}^{i} \in \mathcal{A}_{1} \quad \text { for all } \quad i \in I
$$

as well. Furthermore, the corresponding components $f 1_{C_{1}^{i}}$ and $g 1_{C_{2}^{i}}$ of $f$ and $g$ are measurable. Clearly, the $x_{i}$-section of $\varphi, \varphi\left(x_{i}, \cdot\right)=f\left(x_{i}\right) 1_{C_{2}^{i}}+g 1_{C_{2}^{i}}$ is $\mathcal{A}_{2}$-measurable, hence

$$
g 1_{C_{2}^{i}} \text { is } \mathcal{A}_{2} \text {-measurable and } f 1_{C_{1}^{i}} \text { is } \mathcal{A}_{1} \text {-measurable }
$$

as well.

b) $\mathrm{H}$. Kellerer pointed out to us a very simple and nice argument which yields the stated representation $C=\sum_{i \in I} C_{1}^{i} \times C_{2}^{i}, C_{\nu}^{i} \cap C_{\nu}^{j}=\varnothing$ for $i, j \in I$, $i \neq j$, and $\nu=1,2$ in the proof of Proposition 2.1: The two-dimensional differences of $\varphi_{n}$ disappear. Therefore, given $x_{1}, x_{2} \in E_{1}$ and $y_{1}, y_{2} \in E_{2}$, it follows that $\left(x_{2}, y_{2}\right) \in C$ if $\left(x_{1}, y_{1}\right),\left(x_{1}, y_{2}\right),\left(x_{2}, y_{1}\right) \in C$. This implies that the sections $C_{x_{1}}$ and $C_{x_{2}}$ are either disjoint or coincide, which yields the assertion.

c) $F$ is closed, if $I$ is countable, or if all components $C_{1}^{i} \times C_{2}^{i}$ are one-point sets. But $F$ is not closed in general. The following beautiful counterexample due to N. Gantert (private communication) has surprised us and in fact it pointed out a mistake in a first version of this paper.

E x a m p l e (N. Gantert). Consider $E_{1}=E_{2}=\{0,1\}^{\mathbf{N}}, \mathcal{A}_{1}=\mathcal{A}_{2}=$ $(\mathcal{P}(\{0,1\}))^{\mathbf{N}}$ where $\mathcal{P}(A)$ is the set of all subsets of $A$ and $\mu_{1}=\left(B\left(1, \frac{1}{2}\right)\right)^{\mathbf{N}}$; define the Markov-kernel $K$ from $E_{1}$ to $E_{2}$ by $K(x, \cdot)=\sum_{k=1}^{\infty} \frac{1}{2^{k}} \varepsilon_{x_{(k)}}$, where $x_{(k)}=\left(x_{1}, \ldots, x_{k-1}, x_{k+1}, x_{k+2}, \ldots\right)$, and let $\mu=\mu_{1} \otimes K$ be the product on $E_{1} \times E_{2}$. Furthermore, consider the measurable functions

$$
f_{n}(x)=\sum_{i=1}^{n} x_{i}, \quad g_{n}(y)=-\sum_{i=1}^{n-1} y_{i}, \quad n \geqslant 2 .
$$

Obviously, $f_{n} \rightarrow \infty$ ( $\mu$-a.e. $), g_{n} \rightarrow-\infty(\mu$-a.e. $)$ and

$$
f_{n} \oplus g_{n} \longrightarrow \varphi=\varphi 1_{C} \in L^{0}(\mu) \quad \mu \text {-a.e., }
$$


where $C=\left\{(x, y) \in E ; \lim _{n \rightarrow \infty}\left(f_{n}(x)+g_{n}(y)\right)\right.$ exists $\}$. Clearly, $(x, y) \in C$ if and only if $x_{i+1}=y_{i}$ eventually, and $x, x^{\prime}$ are in the same component if and only if $x_{i}=x_{i}^{\prime}$ eventually, i.e., the components are the atoms of the tail $\sigma$-field on $E_{1}$.

Suppose that $\varphi=f \oplus g$ ( $\mu$-a.s. $)$ for some $f \oplus g \in L^{0}\left(\mu_{1}\right) \oplus L^{0}\left(\mu_{2}\right)$. Let $X=\left(X_{i}, i \in \mathbf{N}\right)=\mathrm{id}_{E_{1}}$ and $X^{k}=\left(X_{i}^{k}, k \in \mathbf{N}\right), X_{i}^{k}=X_{i}$ for $i>k, X_{i}^{k}=0$ for $i \leqslant k . X$ and $X^{k}$ are in the same component, hence $f \circ X-f \circ X^{k}=$ $\lim _{n \rightarrow \infty}\left(f_{n} \circ X-f_{n} \circ X^{k}\right)=\sum_{i=1}^{k} X_{i}$, i.e., $f \circ X=\sum_{i=1}^{k} X_{i}+f \circ X^{k}$. Note that $\sum_{i=1}^{k} X_{i}, f \circ X^{k}$ are independent and that $\sum_{i=1}^{k} X_{i}$ is $B\left(k, \frac{1}{2}\right)$-distributed.

Given $0<\varepsilon \leqslant \frac{1}{20}$, choose $a \in \mathbf{R}$ with $\mu\{|f \circ X|>a\}<\varepsilon$ and choose $b<0$ (by the central limit theorem) such that $\lim _{n \rightarrow \infty} \mu\left\{\sum_{i=1}^{n} X_{i}<\frac{1}{2}(n+b \sqrt{n})\right\}=$ $\frac{1}{8}$, hence $\mu\left\{\sum_{i=1}^{n} X_{i} \geqslant b_{n}\right\}>\frac{3}{4}\left(\forall n \geqslant n_{b}\right), b_{n}:=\frac{1}{2}(n+b \sqrt{n})$. Then, on one hand,

$$
\begin{aligned}
\varepsilon>\mu\{f \circ X>a\} & \geqslant \mu\left\{f \circ X^{n}>a-b_{n}\right\} \mu\left\{\sum_{1}^{n} X_{i} \geqslant b_{n}\right\} \\
& >\frac{3}{4} \mu\left\{f \circ X^{n}>a-b_{n}\right\}
\end{aligned}
$$

yields $\mu\left\{f \circ X^{n}>a-b_{n}\right\}<\frac{4}{3} \varepsilon\left(\forall n \geqslant n_{b}\right)$. On the other hand,

$$
\begin{gathered}
\varepsilon>\mu\{f \circ X \leqslant-a\} \geqslant \mu\left\{f \circ X^{n} \leqslant a-b_{n}\right\} \mu\left\{\sum_{i=1}^{n} X_{i}<b_{n}-2 a\right\} \\
\geqslant\left(1-\frac{4}{3} \varepsilon\right) \frac{1}{16} \quad\left(\forall n \geqslant m_{b} \geqslant n_{b}\right) \text { for some } m_{b} \in \mathbf{N}
\end{gathered}
$$

yields $\varepsilon>\frac{3}{52}$, which contradicts $\varepsilon \leqslant \frac{1}{20}$. Therefore, the limit $\varphi$ having a decomposition in $G$ can not have a decomposition in $F$.

We next state some sufficient conditions which ensure the existence of a measurable decomposition of a limit point $\varphi \in F$, i.e.,

$$
\varphi=f \oplus g \in L^{0}\left(\mu_{1}\right) \oplus L^{0}\left(\mu_{2}\right) .
$$

If $\varphi=f \oplus g 1_{C}=\tilde{f} \oplus \widetilde{g} 1_{C} \in G$ are two different representations in $G$, then $f 1_{C_{1}^{i}}=\tilde{f} 1_{C_{1}^{\mathrm{i}}}+\alpha_{i} 1_{C_{1}^{\mathrm{i}}}$ and $g 1_{C_{2}^{\mathrm{i}}}=\tilde{\mathrm{g}} 1_{C_{2}^{\mathrm{i}}}-\alpha_{i} 1_{C_{2}^{\mathrm{i}}}$ for some $\alpha_{i} \in \mathbf{R}$ and all $i \in I$, i.e., the additive representation of $\varphi$ is unique up to constants on the components $C_{1}^{i} \times C_{2}^{i}$ and we call $\tilde{f}, \tilde{g}$ a modification of $f, g$.

Define the $\mu$-completions of $\mathcal{A}$ and $\mathcal{A}_{i}$,

$$
\begin{aligned}
& \mathcal{A}^{\prime}:=\left(\mathcal{A}_{1} \otimes \mathcal{A}_{2}\right)_{\mu}, \\
& \mathcal{A}_{1}^{\prime}:=\left\{A_{1} \subset E_{1} ; A_{1} \times E_{2} \in \mathcal{A}^{\prime}\right\}, \\
& \mathcal{A}_{2}^{\prime}:=\left\{A_{2} \subset E_{2} ; E_{1} \times A_{2} \in \mathcal{A}^{\prime}\right\} .
\end{aligned}
$$

Then $\mathcal{A}_{1}^{\prime} \otimes \mathcal{A}_{2}^{\prime}$ is the largest product- $\sigma$-algebra in $\mathcal{A}^{\prime}$, and $\mathcal{A}_{i}^{\prime}$ depend on $\mu$, not merely on the narginal measures. $\mu$ extends uniquely to a measure $\mu^{\prime}$ 
on $\mathcal{A}^{\prime}$. Let $\mu_{i}^{\prime}$ denote the marginals of $\mu^{\prime}$ on $\mathcal{A}_{i}^{\prime}$ and

$$
F^{\prime}:=L^{0}\left(\mu_{1}^{\prime}\right) \oplus L^{0}\left(\mu_{2}^{\prime}\right) \text {. }
$$

The proof of the following closedness theorem and proposition shows that centering at the conditional median is the appropriate tool to obtain closedness. We formulate however the theorem with an easy to verify sufficient condition. For the appropriate and nearly necessary but not in general easy to check condition for closedness see the following remarks.

Theorem 2.1. Suppose $\varphi_{n}=f_{n} \oplus g_{n} \in F^{\prime}$ is $\mu^{\prime}$-stochastically convergent to $\varphi$. If

$$
\sup _{n}\left|\varphi_{n}\right| \text { is real-valued, }
$$

then $\varphi$ is in $F^{\prime}$.

$\mathrm{P}$ r o of. We adhere to the notation in the proof of Proposition 2.1. Without loss of generality. $f_{n} \oplus g_{n} \longrightarrow \varphi$ ( $\mu^{\prime}$-a.e.).

1. We can assume that $\sum_{i \in I} C_{1}^{i}=E_{1}$ and $\sum_{i \in I} C_{2}^{i}=E_{2}$. Indeed, by $C \subset \pi_{1}(C) \times E_{2}$, and $\mu^{\prime}(C)=1$ it follows that $\pi_{1}(C) \times E_{2} \in \mathcal{A}^{\prime}$, hence $\pi_{1}(C) \epsilon$ $\mathcal{A}_{1}^{\prime}$ and $\pi_{2}(C) \in \mathcal{A}_{2}^{\prime}$ as well. Thus $\varphi(x, y)=f(x) 1_{\pi_{1}(C)}(x)+g(y) 1_{\pi_{2}(C)}(y)$ ( $\mu^{\prime}$-a.e.) and $E_{1}=\sum_{i \in I} C_{1}^{i}+\left(\pi_{1}(C)\right)^{c}, E_{2}=\sum_{i \in I} C_{2}^{i}+\left(\pi_{2}(C)\right)^{c}$.

2. Define $T_{1}:\left(E_{1}, \mathcal{A}_{1}^{\prime}\right) \longrightarrow(I, J), T_{1}(x)=i$ if $x \in C_{1}^{i}$, and $J$ the $\sigma$-algebra generated by the one-point sets $\{i\}, i \in I$. Let $F_{n}(i, \cdot)$ denote the (right continuous) distribution function of the conditional distribution $\mu^{f_{n} \mid T_{1}=i}$, and let $F_{n}^{-1}(i, \cdot)$ denote the (left continuous) inverse distribution function (the quantile function) of $F_{n}(i, \cdot)$. Then $m_{n}(i):=F_{n}^{-1}\left(i, \frac{1}{2}\right)$ is the smallest conditional median of $f_{n}$ given $T_{1}=i$. Since $F_{n}^{-1}(i, \cdot)$ is left continuous $\forall i \in I$, and $F_{n}^{-1}(\cdot, u)$ is $J$-measurable $\left.\forall u \in\right] 0,1\left[\right.$, we obtain that $F_{n}^{-1}$ is

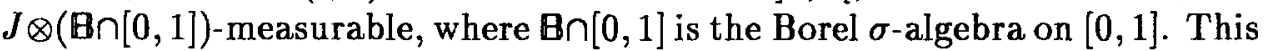
implies that $m_{n}$ is $J$-measurable and, therefore, $m_{n} \circ T_{1}$ is $\sigma\left(T_{1}\right)$-measurable.

3. Let $\left(x_{i}, y_{i}\right) \in C_{1}^{i} \times C_{2}^{i}, i \in I$, be as in the proof of Proposition 2.1. Then

$$
m(i):=\varliminf\left(m_{n}(i)-f_{n}\left(x_{i}\right)\right) \text { is finite } \forall i \in I .
$$

Indeed, if $\psi:=\sup \left|\varphi_{n}\right|$ and $\left(x_{0}, y_{0}\right) \in E$, then by assumption $\mid f_{n}(x)+$ $g_{n}\left(y_{0}\right) \mid \leqslant \psi\left(x, y_{0}\right)$ and $\left|f_{n}\left(x_{0}\right)+g_{n}(y)\right| \leqslant \psi\left(x_{0}, y\right) \quad \forall x \in E_{1}, \quad y \in E_{2}$. Consider the modification $\tilde{f}_{n}(x)=f_{n}(x)-\frac{1}{2}\left(f_{n}\left(x_{0}\right)-g_{n}\left(y_{0}\right)\right), \widetilde{g}_{n}(y)=$ $g_{n}(y)+\frac{1}{2}\left(f_{n}\left(x_{0}\right)-g_{n}\left(y_{0}\right)\right)$. Then $\left|\tilde{f}_{n}\right| \leqslant F:=\psi\left(\cdot, y_{0}\right)+\frac{1}{2} \psi\left(x_{0}, y_{0}\right) \in L^{0}\left(\mu_{1}^{\prime}\right)$, $\left|\tilde{g}_{n}\right| \leqslant G:=\psi\left(x_{0}, \cdot\right)+\frac{1}{2} \psi\left(x_{0}, y_{0}\right) \in L^{0}\left(\mu_{2}^{\prime}\right)$ and $\varphi_{n}=\tilde{f}_{n} \oplus \widetilde{g}_{n}$. Hence we assume without loss of generality that $f_{n}=\tilde{f}_{n}$ and $g_{n}=\tilde{g}_{n}$. Then $\left|m_{n}(i)\right|=$ $\left|\operatorname{med}\left(f_{n} \mid T_{1}=i\right)\right| \leqslant \operatorname{med}\left(F \mid T_{1}=i\right)<\infty$ and $\left|f_{n}\left(x_{i}\right)\right| \leqslant F\left(x_{i}\right)<\infty$ $\forall n \in \mathbf{N}, \forall i \in I$. This yields that $m(i)=\varliminf\left(m_{n}(i)-f_{n}\left(x_{i}\right)\right)$ is finite.

4. Define

$$
\hat{f}(x):=\varlimsup \lim \left(f_{n}(x)-m_{n} \circ T_{1}(x)\right)=f(x)-m \circ T_{1}(x) .
$$


By (2.7) and (2.13) the function $\hat{f}$ is real valued and $\hat{f}$ is $\mathcal{A}_{1}^{\prime}$-measurable, since $f_{n}, m_{n} \circ T_{1}$ are $\mathcal{A}_{1}^{\prime}$-measurable. Define $T_{2}:\left(E_{2}, \mathcal{A}_{2}^{\prime}\right) \longrightarrow(I, J), T_{2}(y)=i$ if $y \in C_{2}^{i}$. Then $m_{n} \circ T_{1}(x)=m_{n} \circ T_{2}(y) \forall(x, y) \in C$. Since

$$
\begin{aligned}
\widehat{\boldsymbol{g}}(y): & =\underline{\underline{\lim }}\left(g_{n}(y)+m_{n}(i)\right) \\
& =\underline{\lim }\left(g_{n}(y)+f_{n}(x)-f_{n}(x)+m_{n}(i)\right) \\
& =\varphi(x, y)-\hat{f}(x) \quad \text { for all }(x, y) \in C_{1}^{i} \times C_{2}^{i}, \\
& \quad \text { and all } i \in I
\end{aligned}
$$

we obtain the $\mathcal{A}_{2}^{\prime}$-measurable function

$$
\widehat{g}(y)=\underline{\lim }\left(g_{n}(y)+m_{n} \circ T_{2}(y)\right) .
$$

and, by (2.14), (2.15), the desired decomposition

$$
\varphi 1_{C}=(\hat{f}+\widehat{g}) 1_{C}, \quad \text { i.e., } \quad \varphi \in L^{0}\left(\mu_{1}^{\prime}\right) \oplus L^{0}\left(\mu_{2}^{\prime}\right) .
$$

Let us point out that if a measurable decomposition of the limit point $\varphi$ in Proposition 2.1 exists at all, then the centering on the conditional median (as in the proof of Theorem 2.1) yields a measurable decomposition. To be precise:

Proposition 2.2. Let $\varphi(x, y) 1_{C}(x, y)=(f(x)+g(y)) 1_{C}(x, y)$ be a limit point of $L^{0}\left(\mu_{1}\right) \oplus L^{0}\left(\mu_{2}\right)$ in $L^{0}(\mu)$ as in Proposition 2.1. If there exists a measurable decomposition $\varphi 1_{C}==\hat{f} \oplus \widehat{g} 1_{C} \in L^{0}\left(\mu_{1}^{\prime}\right) \oplus L^{0}\left(\mu_{2}^{\prime}\right)$, then

$$
\begin{aligned}
\tilde{f} & :=f-\sum_{i \in I} \operatorname{med}\left(f 1_{C_{1}^{i}} \mid T_{1}=i\right) 1_{C_{1}^{i}} \quad \text { is } \mathcal{A}_{1}^{\prime} \text {-measurable, } \\
\widetilde{g}: & =g+\sum_{i \in I} \operatorname{med}\left(f 1_{C_{1}^{i}} \mid T_{1}=i\right) 1_{C_{2}^{i}} \quad \text { is } \mathcal{A}_{2}^{\prime} \text {-measurable, and } \\
\varphi \cdot 1_{C} & =\tilde{f} \oplus \tilde{g} 1_{C},
\end{aligned}
$$

where $T_{1}:\left(E_{1}, \mathcal{A}_{1}\right) \longrightarrow(I, J), T_{1}\left(C_{1}^{i}\right)=i$, and $J$ is generated by the one-point sets.

$\mathrm{P}$ r o of. The additive representation of $\varphi 1_{C}$ is unique up to constants on the components $C_{1}^{i} \times C_{2}^{i} \in \mathcal{A}_{1} \otimes \mathcal{A}_{2}$, hence $\hat{f}=f+\sum_{i \in I} \alpha_{i} 1_{C_{1}^{i}}$ for certain $\alpha_{i} \in \mathbf{R}, i \in I$. Since $f 1_{C_{\mathbf{i}}^{i}}$ is $\mathcal{A}_{\mathbf{1}}$-measurable, we obtain

$$
\begin{aligned}
& \hat{f} 1_{C_{1}^{i}}-\operatorname{med}\left(\hat{f} 1_{C_{1}^{i}} \mid T_{1}=i\right) 1_{C_{1}^{i}} \\
& \quad=f 1_{C_{1}^{i}}+\alpha_{i} 1_{C_{1}^{i}}-\operatorname{med}\left(f 1_{C_{1}^{i}}+\alpha_{i} 1_{C_{1}^{i}}\left|T_{1}=i\right|\right) 1_{C_{1}^{i}} \\
& \quad=f 1_{C_{1}^{i}}-\operatorname{med}\left(f 1_{C_{1}^{i}}\left|T_{1}=i\right|\right) 1_{C_{1}^{i}},
\end{aligned}
$$

and, therefore,

$$
\tilde{f}=\hat{f}-\operatorname{med}\left(\hat{f} \mid T_{1}\right)=f-\sum_{i \in I} \operatorname{med}\left(f 1_{C_{1}^{i}} \mid T_{1}=i\right) 1_{C_{1}^{i}}
$$


is $\mathcal{A}_{1}$-measurable. Define

$$
\widetilde{g}(y):=g(y)+\sum_{i \in I} \operatorname{med}\left(f 1_{C_{1}^{i}} \mid T_{1}=i\right) 1_{C_{1}^{i}}
$$

Then $\widetilde{g}$ is $\mathcal{A}_{2}$-measurable. Indeed, $\widetilde{g}(y) 1_{C}(x, y)=(\varphi(x, y)-\tilde{f}(x)) 1_{C}(x, y)$ is $\mathcal{A}_{1}^{\prime} \otimes \mathcal{A}_{2}^{\prime}$-measurable and for any $\alpha \in \mathbf{R}$ it holds $\left\{\tilde{g} 1_{C} \leqslant \alpha\right\}=E_{1} \times\{\widetilde{g} \leqslant$ $\alpha\} \cap C \subset E_{1} \times\{\tilde{g} \leqslant \alpha\} \subset E_{1} \times\{\tilde{g} \leqslant \alpha\} \cap C+C^{c}$ with $\mu\left(C^{c}\right)=0$. We obtain by definition of $\mathcal{A}_{1}^{\prime}$ that $\{\tilde{g} \leqslant \alpha\} \in \mathcal{A}_{1}^{\prime}$. Hence $\tilde{g}$ is $\mathcal{A}_{2}^{\prime}$-measurable and $\varphi 1_{C}=\tilde{f} \oplus \widetilde{g} 1_{C}$.

Let $\psi$ be a real valued function on $E$, i.e., $\psi \in M(E)$. Then denote by $F_{\psi}^{\prime}$ the subset of $F^{\prime}$ bounded by $\psi$, i.e.,

$$
F_{\psi}^{\prime}=\left\{h \in L^{0}\left(\mu_{1}^{\prime}\right) \oplus L^{0}\left(\mu_{2}^{\prime}\right) ;|h| \leqslant \psi\right\}
$$

Corollary 2.1. $F_{\psi}^{\prime}$ is closed in $L^{0}\left(\mu^{\prime}\right)$ with respect to $\mu^{\prime}$-stochastic convergence.

P r o of. For any limit point $\varphi$ of $F_{\psi}^{\prime}$ there exists a sequence $\varphi_{n}$ in $F_{\psi}^{\prime}$ such that $\varphi_{n} \rightarrow \varphi\left(\mu^{\prime}\right.$-a.e. $)$. Since $\sup _{n}\left|\varphi_{n}\right| \leqslant \psi$, the assertion follows from Theorem 2.1.

Corollary 2.2. Suppose $\left(\varphi_{n}\right)$ is a sequence in $L^{1}\left(\mu_{1}^{\prime}\right) \oplus L^{1}\left(\mu_{2}^{\prime}\right) \subset L^{1}\left(\mu^{\prime}\right)$ and $\left|\varphi_{n}\right| \leqslant \psi \in M(E)$.

a) If $\varphi_{n} \rightarrow \varphi$ in $L^{1}(\mu)$, then $\varphi \in L^{0}\left(\mu_{1}^{\prime}\right) \oplus L^{0}\left(\mu_{2}^{\prime}\right)$. Moreover, $\varphi \in$ $L^{1}\left(\mu_{1}^{\prime}\right) \oplus L^{1}\left(\mu_{2}^{\prime}\right)$ if $\psi\left(\cdot, y_{0}\right) \in L^{1}\left(\mu_{1}^{\prime}\right)$ and $\psi\left(x_{0}, \cdot\right) \in L^{1}\left(\mu_{2}^{\prime}\right)$ for some $\left(x_{0}, y_{0}\right) \in$ $E$.

b) If $\varphi_{n} \rightarrow \varphi$ with respect to the weak topology $\sigma\left(L^{1}\left(\mu^{\prime}\right), L^{\infty}\left(\mu^{\prime}\right)\right)$, then $\varphi \in L^{0}\left(\mu_{1}^{\prime}\right) \oplus L^{0}\left(\mu_{2}^{\prime}\right)$.

$\mathrm{P}$ r o o f. a) follows from Theorem 2.1. The integrability follows from majorized convergence.

b) follows from a) observing that $\left(L^{1}\left(\mu_{1}^{\prime}\right) \oplus L^{1}\left(\mu_{2}^{\prime}\right)\right) \cap\left\{h \in L^{1}\left(\mu^{\prime}\right)\right.$; $|h| \leqslant \psi\}$ is convex and, therefore, the weak and the norm-closure of this set coincide.

R e m a r k s. a) The limit $\varphi$ in Corollary 2.2 a) is not in $L^{1}\left(\mu_{1}^{\prime}\right) \oplus L^{1}\left(\mu_{2}^{\prime}\right)$ in general (cf. [18]).

b) An inspection of the proof of Theorem 2.1 shows that the boundedness condition (2.12) is only used to prove the finiteness condition (2.13), which is violated in the counterexample. So we can reformulate Theorem 2.1 in a more general form substituting (2.12) by (2.13), but in general it seems to be difficult to check the latter condition directly. By Proposition 2.2 it is seen that condition (2.13) is «nearly» necessary, too.

c) Let $L_{++}^{0}\left(\mu_{i}^{\prime}\right):=\left\{f \in L^{0}\left(\mu_{i}^{\prime}\right) ; f>0\left(\mu_{i}^{\prime}\right.\right.$-a.e. $\left.)\right\}$ and define the product $f \otimes g(s, t):=f(s) g(t)$. Then the limit points of sequences in $L_{++}^{0}\left(\mu_{1}^{\prime}\right) \otimes$ $L_{++}^{0}\left(\mu_{2}^{\prime}\right)$ bounded in $L_{++}^{0}\left(\mu^{\prime}\right)$ are in $L_{++}^{0}\left(\mu_{1}^{\prime}\right) \otimes L_{++}^{0}\left(\mu_{2}^{\prime}\right)$ again. The proof is immediate from Theorem 2.1 by using logarithms. 
d) One might think that it is possible to get a more direct construction of a measurable representation of a limit point $\varphi(x, y) \in L^{1}(\mu)$ in the form $f(x)+g(y)$ by means of the optimal $L^{1}$-approximation operators $S_{i}$ with respect to $L^{1}\left(\mu_{i}\right)$, i.e., the conditional medians

$$
S_{1} \varphi(x)=\operatorname{med}_{\mu}\left(\varphi \mid \pi_{1}=x\right), \quad S_{2} \varphi(y)=\operatorname{med}_{\mu}\left(\varphi \mid \pi_{2}=y\right)
$$

(cf. [19]). Consider the following example:

$$
\begin{aligned}
E_{1} & =E_{2}=\{0,1\}, \quad 0<\varepsilon<\frac{1}{4}, \\
\mu & :=\left(\frac{1}{2}-\varepsilon\right)\left(\delta_{\{(0,0)\}}+\delta_{\{(1,1)\}}\right)+\varepsilon\left(\delta_{\{(0,1)\}}+\delta_{\{(1,0)\}}\right)
\end{aligned}
$$

and $\varphi(x, y):=f(x)+g(y)$ with $f(0)=g(0)=0, f(1)=-g(1)=1$. Then

$$
\begin{aligned}
& S_{1} \varphi(x)=\operatorname{med}_{\mu}\left(\varphi \mid \pi_{1}=x\right)=0 \quad \text { and } \\
& S_{2} \varphi(y)=\operatorname{med}_{\mu}\left(\varphi \mid \pi_{2}=y\right)=0 .
\end{aligned}
$$

In particular, the alternating projection algorithm does not converge to an optimal approximation and it seems impossible to identify $f, g$ in terms of $S_{1}, S_{2}$. The optimal approximation is given by the projection equations

$$
S_{1}(\varphi-g)=f, \quad S_{2}(\varphi-f)=g .
$$

But it is not clear how to solve them.

Theorem 2.1 shows that the limit point in $L^{0}\left(\mu^{\prime}\right)$ of a sequence $f_{n} \oplus g_{n}$ in $F^{\prime}$ satisfying (2.12) or (2.13) is of the form $f \oplus g$, where $f, g$ are measurable only with respect to the $\mu$-completions $\mathcal{A}_{i}^{\prime}$. Therefore, let us point out that even in fairly general situations equality $\mathcal{A}_{i}^{\prime}=\mathcal{A}_{i}$ holds if $\mathcal{A}_{i}$ are assumed to be complete in the usual sense. Concerning the notion of perfect measure spaces we refer to [16, Definition 2.1].

Theorem 2.2. If $\left(E_{i}, \mathcal{A}_{i}, \mu_{i}\right)$ are complete perfect measure spaces, then $\mathcal{A}_{i}=\mathcal{A}_{i}^{\prime}$. Therefore, given $\psi \in M(E)$, the set $F_{\psi} \subset\left(L^{0}\left(\mu_{1}\right) \oplus L^{0}\left(\mu_{2}\right)\right)$ is closed in $L^{0}(\mu)$.

$\mathrm{P}$ r o of. A basic result in perfect measure spaces (cf. [16, Theorem 12.2.1]) implies that $\mu_{*}\left(A_{1} \times E_{2}\right)=\left(\mu_{1}\right)_{*}\left(A_{1}\right)$ for any $A_{1} \subset E_{1}$, where $\mu_{*},\left(\mu_{1}\right)_{*}$ are the inner measures of $\mu, \mu_{1}$. Given $A_{1} \in \mathcal{A}_{1}^{\prime},\left(\mu_{1}\right)_{*}\left(A_{1}\right)+$ $\left(\mu_{1}\right)_{*}\left(A_{1}^{c}\right)=\mu_{*}\left(A_{1} \times E_{2}\right)+\mu_{*}\left(A_{1}^{C} \times E_{2}\right)=\mu^{\prime}\left(A_{1} \times E_{2}\right)+\mu^{\prime}\left(A_{1}^{c} \times E_{2}\right)=1$. Hence, there are $B_{i} \in \mathcal{A}_{1}, B_{1} \subset A_{1}, B_{2} \subset A_{1}^{c}$ with $\mu_{1}\left(B_{1}\right)+\mu_{1}\left(B_{2}\right)=1$. Therefore, $B_{1} \subset A_{1} \subset B_{2}^{c}$ and $\mu_{1}\left(B_{1}\right)=\mu\left(B_{2}^{c}\right)$. This implies $A_{1} \in \mathcal{A}_{1}$, since $\mathcal{A}_{1}$ is $\mu_{1}$-complete.

$\mathrm{R} \mathrm{e} \mathrm{m}$ a $\mathrm{rk}$. Note that perfectness of a measure space is preserved by completion (cf. $[16,2.2$, p. 5]). In particular, Theorem 2.2 implies the closedness property of $F_{\psi}$ for polish spaces and, more general; for analytic spaces. The necessity to enlarge $\mathcal{A}_{i}$ to $\mathcal{A}_{i}^{\prime}$ in Theorem 2.1 arises from the fact that 
projections of product measurable sets onto the marginal spaces are known to be in the completions of the marginal spaces only under additional assumptions like analyticity. Therefore, we conjecture that the enlargement of $\mathcal{A}_{i}$ to $\mathcal{A}_{i}^{\prime}$ in Theorem 2.1 can not be omitted in general.

\section{On the Generalized Schrödinger Problem}

Let $M\left(\mu_{1}, \mu_{2}\right)$ be the set of all probability measures on $\left(E_{1} \times E_{2}, \mathcal{A}_{1} \otimes\right.$ $\mathcal{A}_{2}$ ) with marginals $\mu_{i}$. Let $I(\nu \mid \mu)=\int \ln (d \nu / d \mu) d \nu$ denote the KullbackLeibler distance and define $\nu^{*} \in M\left(\nu_{1}, \nu_{2}\right)$ to be the $I$-projection of $\mu$ on $M\left(\nu_{1}, \nu_{2}\right)$ if

$$
I\left(\nu^{*} \mid \mu\right)=\inf \left\{I(\nu \mid \mu) ; \nu \in M\left(\nu_{1}, \nu_{2}\right), \nu \ll \mu\right\}=: m .
$$

From the closedness results in Section 2 we obtain the existence of a solution of the generalized Schrödinger equation (1.1), (1.3), if $m$ is finite.

Theorem 3.1. Assume that $\nu_{i} \ll \mu_{i}, i=1,2$, and $m<\infty$.

a) There exists a uniquely determined I-projection $\nu^{*}$ on $M\left(\nu_{1}, \nu_{2}\right)$.

b) There exists $a \otimes b \in L_{+}^{0}\left(\nu^{*}\right)$ such that $\left(d \nu^{*} / d \mu\right)(x, y)=a(x) b(y)$, $\left(\nu^{*}\right.$-a.e. $)$.

P r o of. a) $M\left(\nu_{1}, \nu_{2}\right)$ is closed in variation norm and so the assertion follows from [5, Theorem 2.1].

b) By Theorem 3.1 of $[5], \ln \left(d \nu^{*} / d \mu\right)$ belongs to the closure of $L^{1}\left(\nu_{1}\right) \oplus$ $L^{1}\left(\nu_{2}\right)$ in $L^{1}\left(\nu^{*}\right)$. By Proposition 2.1, therefore, $\ln \left(d \nu^{*} / d \mu\right)(x, y)=f(x)+$ $g(y)\left(\nu^{*}\right.$-a.e.) for some $f \oplus g \in G$ and the result follows with $a=\exp f$, $b=\exp g$.

$\mathrm{R}$ e $\mathrm{m}$ a $\mathrm{rks}$. a) The functions $a, b$ are not unique in general, but only the product $a \otimes b$ is unique $\nu^{*}$-a.e. It is easily seen that $a$ and $b$ are unique up to constants if and only if any two points $\left(x^{\prime}, y^{\prime}\right)$ and $\left(x^{\prime \prime}, y^{\prime \prime}\right)$ in the support $A$ of $\nu^{*}$ are finitely connected in $A$, i.e., there are finitely many points $\left(x_{1}, y_{1}\right):=\left(x^{\prime}, y^{\prime}\right),\left(x_{2}, y_{2}\right), \ldots,\left(x_{n}, y_{n}\right):=\left(x^{\prime \prime}, y^{\prime \prime}\right)$ in $A$, such that $x_{i}=x_{i+1}$ or $y_{i}=y_{i+1}$ for all $i=1, \ldots, n-1$. For example, this is true if $\mu=h \mu_{1} \otimes \mu_{2}, h>0, \mu_{1} \otimes \mu_{2}$-a.e.

b) Theorem 3.1 is of importance for the derivation of characterizations and properties of (generalized) Schrödinger bridges. To be precise, let $P_{0}$ be the Wiener measure on $S:=C\left([0, T], \mathbf{R}^{d}\right), 0<T<\infty$. Furthermore, with $\Omega=S^{\mathbf{N}}$ and with $P=P_{0}^{\mathbf{N}}$ let $X_{i}$ be the projections. Föllmer [7] noted that Schrödinger bridges have a large deviation interpretation. If $\mu_{n}:=\frac{1}{n} \sum_{i=1}^{n} \delta_{X_{i}}$ is the empirical measure of $X_{1}, \ldots, X_{n}$, then, by Sanov's large deviation theorem, $\left(\mu_{n}\right)$ satisfies a $L D$-principle with rate function $I\left(Q_{0} \mid P_{0}\right)$.

For a subset $\Gamma$ of probability measures on $S$ it holds:

$$
P\left\{\mu_{n} \in \Gamma\right\} \sim \exp \left\{-n \inf _{Q_{0} \in \Gamma} I\left(Q_{0} \mid P_{0}\right)\right\} .
$$


If $\mu=P_{0}^{(X(0), X(T))}$ is the joint distribution of $(X(0), X(T))$ with respect to Wiener measure and

$$
\Gamma:=\left\{Q_{0} \in M^{1}(S) ; Q_{0}^{X_{0}}=\nu_{1}, Q_{0}^{X(T)}=\nu_{2}\right\}
$$

with $\nu_{i} \ll \mu_{i}$, then $\Gamma$ describes large deviations at the initial and final time points.

For any $Q_{0} \in \Gamma$ it holds

$$
I\left(Q_{0} \mid P_{0}\right)=I(\nu \mid \mu)+\int I\left(Q_{x}^{y} \mid P_{x}^{y}\right) \nu(d x, d y),
$$

where $\nu=Q_{0}^{(X(0), X(T))}$ and $Q_{x}^{y}, P_{x}^{y}$ are the conditional distributions of $Q_{0}, P_{0}$ given $X(0)=x, X(T)=y$. This implies

$$
I\left(Q_{0} \mid P_{0}\right) \geqslant I(\nu \mid \mu) \geqslant I\left(\nu^{*} \mid \mu\right)=I\left(P^{*} \mid P_{0}\right),
$$

where $P^{*}:=\int P_{x}^{y} \nu^{*}(d x, d y)$ is the Schrödinger bridge. This minimum entropy interpretation of $P^{*}$ yields via Sanov's theorem (3.2) that $P^{*}$ is «favoured» in the sense of large deviations by the empirical distribution in $\Gamma$.

Related results can be derived for modifications of $\Gamma$ (like Prokhorovtype neighbourhoods) and also for general $P_{0}$, replacing the Wiener measure, and thus leading to generalized Schrödinger bridges $P^{*}$. For the ample literature on these kind of problems we refer to [7], [14], [6], [4], [2], [20], $[3],[11]$.

The factorization property $\left(d \nu^{*} d \mu\right)(x, y)=a(x) b(y),\left(\nu^{*}\right.$-a.e. $)$ is crucial for the derivation of characteristic properties of the Schrödinger bridges (like the (reciprocal) Markov property, diffusion property, $h$-path process). Theorem 3.1 gives a product form of the density but in general with nonmeasurable factors $a, b$ only. It is however not clear whether this factorization is enough to derive the above mentioned properties of Schrödinger bridges.

\section{Some Extensions and Applications}

Clearly, the statements in Section 2 carry over to product spaces of $k \in \mathbf{N}$ factors. In particular, let $(E, \mathcal{A})=\otimes_{i=1}^{k}\left(E_{i}, \mathcal{A}_{i}\right)$, let $\mu$ be a probability measure on $(E, \mathcal{A})$ with marginals $\mu_{i}$ and $\psi \in M(E)$.

Proposition 4.1. a) $\left(\oplus_{i=1}^{k} M\left(E_{i}\right)\right) \cap L^{0}\left(\mu^{\prime}\right)$ is closed in the metric space $L^{0}\left(\mu^{\prime}\right)$ with respect to $\mu^{\prime}$-stochastic convergence.

b) $\left(\oplus_{i=1}^{k} L^{0}\left(\mu_{i}^{\prime}\right)\right)_{\psi}$ is closed in $L^{0}\left(\mu^{\prime}\right)$.

For another aspect let $(\Omega, \mathcal{B}, P)$ be a complete probability space. Let $\mathcal{B}_{1}, \ldots, \mathcal{B}_{k} \subset \mathcal{B}$ be sub- $\sigma$-algebras of $\mathcal{B}$ containing the $P$-null sets. $P_{i}:=P \mid \mathcal{B}_{i}$ denote the restriction of $P$ to $\mathcal{B}_{i}$ and $L^{0}\left(P_{i}\right)=L^{0}\left(\Omega, \mathcal{B}_{i}, P_{i}\right)$ are the realvalued $\mathcal{B}_{i}$-measurable functions. Define $\mu$ on $\otimes_{i=1}^{k}\left(\Omega, \mathcal{B}_{i}\right)$ by $\mu\left(B_{1} \times \cdots \times\right.$ $\left.B_{k}\right)=P\left(B_{1} \cap \cdots \cap B_{k}\right)$ and let $T_{i}:(\Omega, \mathcal{B}) \longrightarrow\left(\Omega, \mathcal{B}_{i}\right), T_{i}=\mathrm{id}_{\Omega}$. Note that 
$P^{\left(T_{1}, \ldots, T_{k}\right)}=\mu, P^{T_{i}}=P_{i}=\mu_{i}$, and that $\mathcal{B}_{i}$ coincides with its $\mu$-completion.

This leads to the following

Proposition 4.2. $\left(\oplus_{i=1}^{k} L^{0}\left(P_{i}\right)_{\psi}\right)$ is closed in $L^{0}(P)$ for any $\psi \in M(\Omega)$.

P r o of. Let $\varphi$ be a limit point and $\varphi_{n}=\sum_{i=1}^{k} h_{n i}$ be a sequence in $\left(\oplus_{i=1}^{k} L^{0}\left(P_{i}\right)_{\psi}\right)$ such that $\varphi_{n} \rightarrow \varphi(P$-a.e. $)$. Clearly, $h_{n i}$ are $\sigma\left(T_{i}\right)=\mathcal{B}_{i^{-}}$ measurable and $\varphi$ is $\sigma\left(T_{1}, \ldots, T_{k}\right)=\sigma\left(\cup_{i=1}^{k} B_{i}\right)$-measurable. By the factorization theorem, there are measurable functions $k_{n i}:\left(\Omega, \mathcal{B}_{i}\right) \longrightarrow(\mathbf{R}, \mathbf{B})$ and $k:\left(\Omega^{k}, \otimes_{i=1}^{k} \mathcal{B}_{i}\right) \longrightarrow(\mathbf{R}, \mathbf{B})$ such that $k_{n i} \circ T_{i}=h_{n i}$ and $k \circ\left(T_{1}, \ldots, T_{k}\right)=\varphi$. This yields $1=P\left\{\lim _{n \rightarrow \infty} \sum_{i=1}^{k} h_{n i}=\varphi\right\}=\mu\left\{\lim _{n \rightarrow \infty} \sum_{i=1}^{k} k_{n i}=k\right\}$, since $P^{\left(T_{1}, \ldots, T_{k}\right)}=\mu$. By assumption, $\left|\sum_{i=1}^{k} k_{n i}\left(\omega_{i}\right)\right| \leqslant \sum_{i=1}^{k} \psi\left(\omega_{i}\right)<$ $\infty$ for all $\left(\omega_{1}, \ldots, \omega_{k}\right) \in \Omega^{k}$, hence Theorem 2.1 or Proposition 4.1 yields $k\left(\omega_{1}, \ldots, \omega_{k}\right)=\sum_{i=1}^{k} k_{i}\left(\omega_{i}\right)$ for some $k_{i} \in L^{0}\left(\mu_{i}\right)=L^{0}\left(P_{i}\right)$. This implies $\varphi=\sum_{i=1}^{k} h_{i}$, (P-a.e.), where $h_{i}=k_{i} \circ T_{i}$.

$\mathrm{R} \mathrm{e} \mathrm{m} \mathrm{a} \mathrm{r} \mathrm{k.} \mathrm{In} \mathrm{particular,} \mathrm{Proposition} 4.2$ applies to functions of several variables like $\sum_{i=1}^{k-1} f\left(x_{i}, x_{i+1}\right)$ or as in ridge regression theory to functions of the type $\sum_{i=1}^{k} f_{i}\left(A_{i} x\right)$, where $A_{i}$ are some fixed functions on $E$.

From Theorem 3.1 and these propositions we obtain an interesting structure theorem for distributions with multivariate marginals. Let $(E, \mathcal{A})=$ $\otimes_{i=1}^{k}\left(E_{i}, \mathcal{A}_{i}\right)$, let $\mathcal{H} \subset \mathcal{P}(\{1, \ldots, k\})$ be a system of subsets of $\{1, \ldots, k\}$ and let $\mu \in M^{1}(E, \mathcal{A})$ have $H$-marginals $\mu_{H}:=\mu^{\pi_{H}}, H \in \mathcal{H}$, where $\pi_{H}$ : $E \longrightarrow \prod_{j \in H} E_{j}=: E_{H}$ is the projection on the $H$-component. Let $\nu_{H} \in$ $M^{1}\left(E_{H}, \mathcal{A}_{H}\right), \mathcal{A}_{H}:=\otimes_{j \in H} \mathcal{A}_{j}$, be continuous with respect to $\mu_{H}, H \in \mathcal{H}$, and let $M_{\mathcal{H}}:=M\left(\left(\nu_{H}\right), H \in \mathcal{H}\right)$ be the set of all probabilities on $(E, \mathcal{A})$ with $H$-marginals $\nu_{H}, H \in \mathcal{H}$. The following proposition gives an interesting relation between $M\left(\mu_{H}, H \in \mathcal{H}\right)$ and $M\left(\nu_{H}, H \in \mathcal{H}\right)$.

Proposition 4.3. If $M_{\mathcal{H}}:=M\left(\nu_{H}, H \in \mathcal{H}\right) \neq \varnothing$ and $\inf \{I(\nu \mid \mu) ; \nu \in$ $\left.M_{\mathcal{H}}\right\}<\infty$, then there exists $\otimes_{H \in \mathcal{H}^{\prime}} a_{H} \in L_{+}^{0}(\mu)$ such that $\nu^{*}=\left(\otimes_{H \in \mathcal{H}^{\prime}} a_{H}\right) \mu \epsilon$ $M_{\mathcal{H}}$, where $\otimes_{H \in \mathcal{H}} a_{H}(x)=\prod_{H \in \mathcal{H}} a_{H}\left(x_{H}\right), x_{H}:=\pi_{H}(x)$.

$\mathrm{P}$ r o o f. The proof is similar to that of Theorem 3.1 applying Proposition 4.1 with sub- $\sigma$-algebras $\sigma\left(\pi_{H}\right) \subset \mathcal{A}=\otimes_{j=1}^{n} \mathcal{A}_{j}, H \in \mathcal{H}$.

The following proposition gives a basic existence result for additive statistical models. Consider $(E, \mathcal{A})=\otimes_{i=1}^{k}\left(E_{i}, \mathcal{A}_{i}\right)$ and a probability measure $\mu$ on $(E, \mathcal{A})$ with marginals $\mu_{i}$. In additive statistical models the systematic part of the observations is assumed to be in $F^{\alpha}:=\oplus_{i=1}^{k} L^{\alpha}\left(\mu_{i}\right) \subset L^{\alpha}(\mu)$, where $0<\alpha \leqslant \infty$.

Proposition 4.4. a) For any $0<\alpha \leqslant \infty$ there exists a probability space $(E, \mathcal{A}, \mu)$ such that $F^{\alpha}$ is not closed in $L^{\alpha}(\mu)$.

b) For $1<\alpha<\infty$ and any $\varphi \in L^{\alpha}(\mu)$ there exists a unique optimal approximation $\bar{\varphi}$ of $\varphi$ in the closure of $F^{\alpha}$ in $L^{\alpha}(\mu)$ and $\bar{\varphi} \in\left(\oplus_{i=1}^{k} M\left(E_{i}\right)\right)$ ก $L^{0}\left(\mu^{\prime}\right)$.

P r o of. a) If $0<\alpha<\infty$, then consider $E_{1}=E_{2}=\mathbf{N}$ and probabilities $\mu_{1}=\mu_{2}$ with density $p(2 n-1)=p(2 n)=\frac{1}{2} 2^{-n}$ and the probability measure $\mu \ll \mu_{1} \otimes \mu_{2}$ with density $q(2 n, 2 n-1)=q(2 n-1,2 n)=\frac{1}{2} 2^{-2 n}$, 
$q(2 n, 2 n)=q(2 n-1,2 n-1)=\frac{1}{2}\left(2^{-n}-2^{-2 n}\right) . \mu$ has marginals $\mu_{1}, \mu_{2}$. Define $f, g: \mathbf{N} \longrightarrow \mathbf{R}^{1}, f(2 n-1)=2^{n / \alpha}, f(2 n)=0$, and $g(2 n-1)=-2^{n / \alpha}$, $g(2 n)=0$. Then $f \notin L^{\alpha}\left(\mu_{1}\right), g \notin L^{\alpha}\left(\mu_{2}\right)$ but $\varphi=f \oplus g \in L^{\alpha}(\mu)$ and $\int|\varphi|^{\alpha} d \mu=1$. With $f_{n}:=f 1_{\{1, \ldots, n\}}, g_{n}:=g 1_{\{1, \ldots, n\}}, f_{n} \in L^{\alpha}\left(\mu_{1}\right), g_{n} \in$ $L^{\alpha}\left(\mu_{2}\right)$ and $\varphi_{n}:=f_{n} \oplus g_{n} \longrightarrow \varphi$ in $L^{\alpha}(\mu)$. Therefore, $\varphi$ is in the closure of $F^{\alpha}$ in $L^{\alpha}(\mu)$. For any representation $\varphi=f^{\prime} \oplus g^{\prime}(\mu$-a.e. $)$, it holds $f^{\prime}(i)=f(i)+c_{i}$, $g^{\prime}(j)=g(j)-c_{j}$ for some constants $c_{i}$ and $(i, j)$ with $q(i, j)>0$. With $d_{n}:=c_{2 n-1}=-c_{2 n}$ and using the inequality $|a+b|^{\alpha} \leqslant 2^{\alpha}\left(|a|^{\alpha}+|b|^{\alpha}\right)$ we obtain $\int\left|f^{\prime}\right|^{\alpha} d \mu_{1}=\sum_{n=1}^{\infty}\left[\left|2^{n / \alpha}+d_{n}\right|^{\alpha} \cdot \frac{1}{2} \cdot 2^{-n}+\left|-d_{n}\right|^{\alpha} \frac{1}{2} 2^{-n}\right]=\infty$. This implies that $\varphi$ is not in $F^{\alpha}$.

If $\alpha=\infty$, then consider $E_{1}=E_{2}=\mathbf{R}^{1}, \mathcal{A}_{1}=\mathcal{A}_{2}=\mathcal{B}^{1}$. Let $Q_{1}=$ $Q_{2}=N(0,1), A=\{(x, y) ; x-1 \leqslant y \leqslant x+1\} \in \mathcal{B}^{2}$ and let $\mu$ be the product $Q_{1} \otimes Q_{2}$ conditional on $A$. Furthermore, let $f=\mathrm{id}_{\mathbf{R}^{1}}, g=-\mathrm{id}_{\mathbf{R}^{1}}$ and $\varphi:=f \oplus g . \varphi=f \oplus g$ is the unique measurable additive decomposition of $\varphi$ but $f \notin L^{\infty}\left(\mu_{1}\right), g \notin L^{\infty}\left(\mu_{2}\right), \varphi \in L^{\infty}(\mu)$, since $|\varphi| \leqslant 1$ ( $\mu$-a.e.).

b) Let $\left(\varphi_{n}\right) \subset F^{\alpha}$ be a sequence such that $\left\|\varphi-\varphi_{n}\right\|_{\alpha} \longrightarrow \inf \left\{\|\varphi-h\|_{\alpha}\right.$; $\left.h \in F^{\alpha}\right\}=: a$. Then $\left(\varphi_{n}\right)$ is norm-bounded and, therefore, relatively weakly sequentially compact. Since $F^{\alpha}$ is convex the norm-closure and the weak closure coincide and, therefore, $\|\varphi-\bar{\varphi}\|=a$ for any accumulation point $\bar{\varphi}$ of $\left(\varphi_{n}\right)$. Obviously, $\bar{\varphi}$ is uniquely determined and by Proposition 4.1, $\bar{\varphi} \in\left(\oplus_{i=1}^{k} M\left(E_{i}\right)\right) \cap L^{0}(\mu)$ and $\bar{\varphi}$ is the optimal approximation of $\varphi$.

The final application will be an extension of a famous representation theorem of Kolmogorov (1957) for continuous functions $f:[0,1]^{n} \longrightarrow \mathbf{R}^{1}$ of $n$ variables, disproving conjecture no. 13 of Hilbert's list of 23 problems. Kolmogorov's theorem states the existence of real continuous functions $\alpha_{i j}:[0,1] \longrightarrow \mathbf{R}^{1}, 1 \leqslant i \leqslant 2 n+1,1 \leqslant j \leqslant n$, such that any continuous function $f=f\left(x_{1}, \ldots, x_{n}\right)$ has a representation of the form

$$
f\left(x_{1}, \ldots, x_{n}\right)=\sum_{i=1}^{2 n+1} g\left(\sum_{j=1}^{n} \alpha_{i j}\left(x_{j}\right)\right)
$$

with some continuous functions $g$ depending on $f$. The functions $\alpha_{i j}$ even may be chosen to be Lipschitz-continuous, and $\left(\sum_{j} \alpha_{i j}\right)$ to be one-to-one. As reference for this result and several modifications we refer to Chapter 11 of [13]. (4.1) is considered to be a theoretical justification of neural networks. We obtain a «generalization» to measurable functions as follows.

Proposition 4.5. Let $\mu \in M^{1}\left(\mathbf{R}^{n}, B^{n}\right)$ and let $f \in L^{0}(\mu)$ be locally bounded. Then

$$
f\left(x_{1}, \ldots, x_{n}\right)=\sum_{i=1}^{2 n+1} g\left(\sum_{j=1}^{n} \alpha_{i j}\left(x_{i}\right)\right) \quad(\mu \text {-a.e. })
$$

for some measurable functions $g: \mathbf{R}^{1} \longrightarrow \mathbf{R}^{1}$ and $\alpha_{i j}: \mathbf{R}^{1} \longrightarrow \mathbf{R}^{1}$ piecewise Lipschitz-continuous. 
$\mathrm{P}$ r o of. Since $f$ is assumed to be locally bounded on $\mathbf{R}^{n}$, it is majorized by a continuous function $h$ on $\mathbf{R}^{n},|f| \leqslant h$. Let without loss of generality $f \geqslant 0$, then decomposing $\mathbf{R}^{n}$ into disjoint unions of cubes we can approximate $f$ by a sequence $0 \leqslant f_{m} \leqslant n$ of continuous functions with respect to $\mu$-stochastic convergence. By (4.1) $f_{m}$ has a representation of the form

$$
f_{m}(x)=\sum_{i=1}^{2 n+1} g_{m}\left(\sum_{j=1}^{n} \alpha_{i j}\left(X_{j}\right)\right),
$$

where $g_{m}$ are continuous on the cubes and, in particular, measurable on $\mathbf{R}^{n}$ and the $\left(\alpha_{i j}\right)$ are as in (4.1). From the proof of (4.1) in [14] it is seen that a representing function $g=g_{f}$ in (4.1) can be chosen such that $f_{1} \leqslant f_{2}$ implies $g_{f_{1}} \leqslant g_{f_{2}}$. This yields $0 \leqslant g_{m} \leqslant g_{h}$ for all $m \in \mathbf{N}$. Then, by Proposition 4.2 with $\mathcal{B}_{i}=\sigma\left(T_{i}\right)$, Remark b) implies the existence of a measurable function $g$ such that $f(x)=\sum_{i=1}^{2 n+1} g\left(\sum_{j=1}^{n} \alpha_{i j}\left(x_{j}\right)\right)$ ( $\mu$-a.e.).

$\mathrm{R}$ e m a r k s. a) Kolmogorov's theorem is considered as a theoretical foundation of neural networks (cf. [9, p. 122-137], [17]). There are many papers on generalizations of this theorem to non continuous functions and to variants of the theorem with special kind of functions $\alpha_{i j}$ in the literature on neural networks. Proposition 4.5 improves the known representation and approximation results for neural networks like the back propagation theorem (cf. [9, p. 132] and references given there). For the first time it states an exact representation result for standard feed forward networks with one hidden layer. It was known up to now that two hidden layers are sufficient (cf. the discussion of this point in [17] and [9]).

b) By the monotonicity property of the representing function $g$ in (4.1) it is clear that the representation (4.1) holds for any continuous function $f$ on $\mathbf{R}^{n}$. Therefore, it is possible to extend the representation property to measurable functions majorized on open subsets $U_{j}$ by continuous functions $f_{j}$ such that $\mu\left(\partial U_{j}\right)=0,\left(U_{j}\right)$ pairwise disjoint and $\cup \overline{U_{j}}=\mathbf{R}^{n}$.

Acknowledgement. We are grateful to H. Föllmer and H. Kellerer for critical and stimulating remarks on this paper. N. Gantert informed us on the counterexample in Section 2.

\section{REFERENCES}

1. Beurling A. An automorphism of product measures. - Ann. Math., 1960, v. 72, p. 189-200.

2. Blaquiere A., Sigal-Pouchard M. Schrödinger processes with unbounded or singular potentials, conditional Sanov property. - Proceedings of 5th Workshop of the Bellman Continuum, Hawai, 1993.

3. Bolthausen E. Markov process large deviations in $\tau$-topology. - Stoch. Process. Appl., 1987, v. 25, p. 95-108.

4. Brunaud $M$. Finite Kullback information diffusion laws with fixed marginals and 
associated large deviations functionals. - Stoch. Process. Appl., 1993, v. 44, p. 329345 .

5. Csiszar $I$. $I$-divergence geometry of probability distributions and minimization problems. - Ann. Probab., 1975, v. 3, p. 146-158.

6. Dawson D., Gorostiza L., Wakolbinger A. Schrödinger processes and large deviations. - J. Math. Phys., 1990, v. 31, p. 2385-2388.

7. Föllmer $H$. Random fields and diffusion processes. - Lect. Notes Math., 1988, v. 1362, p. 101-203.

8. Fortet $P$. R. Résolution d'un système d'équations de M. Schrödinger. - J. Math. Pures Appl., 1940, v. 9, p. 83-105.

9. Hecht-Nielsen R. Neurocomputing. Reading, MA: Addison-Wesley, 1989.

10. Hobby $C$., Pyke R. Doubly stochastic operators obtained from positive operators. Pac. J. Math., 1965, v. 15, p. 153-157.

11. Jamison B. Reciprocal processes. - Z. Wahrscheinlichkeitsheor. verw. Geb., 1974, B. 30, S. 65-86.

12. Колмогоров A. Н. О представлении непрерывных функций нескольких переменных суперпозициями непрерывных функций меныпего числа переменных. Докл. АН СССР, т. 108, с. 179-182.

13. Light W. A., Cheney E. W. Approximation Theory in Tensor Product Spaces. (Lect. Notes Math., v. 1169). Berlin: Springer-Verlag, 1985.

14. Lorentz G. G. Approximation of Functions. New York: Holt, Rinehart and Winston, 1966.

15. Nagasawa $M$. Can the Schrödinger equation be a Boltzmann equation? - In: Diffusion Processes and Related Problems in Analysis/Ed. by M. Pinsky. Basel: Birkhäuser, 1990.

16. Ramachandran D. Perfect Measures. New York: Macmillan, 1979.

17. Ripley B. D. Statistical Aspects of Neural Networks. (Monographs on Statist. Appl. Probab. 50.) London: Chapman \& Hall, 1993, p. 40-123.

18. Rüschendorf $L$., Thomsen $W$. Note on the Schrödinger equation and $I$-projections. Statist. Probab. Lett., 1993, v. 17, p. 369-375.

19. Shintani $T$., Ando $T$. Best approximation in $L^{1}$-space. $-\mathrm{Z}$. Wahrscheinlichkeitstheor. verw. Geb., 1975, B. 33, S. 33-39.

20. Schrödinger E. Über die Umkehrung der Naturgesetze. Sitzungsberichte Preuss. Akad. Wiss. Berlin, Phys. Math., 1931, B. 144, S. 144-153.

21. Wakolbinger A. Schrödinger bridges from 1931 to 1991. - In: Proc. 4th Latin America Congress in Probab. Math. Statist./Ed. by E. Cabana, 1992, p. 61-79.

Поступила в редакщию 10.II.1995 